\title{
MULTICOMPONENT INTERVENTIONS AGAINST FRAILTY
}

\author{
D. Azzolino ${ }^{1,2}$, M. Cesari ${ }^{1,2}$
}

\section{Dear Editor,}

The prevention and management of frailty imply the delay of functional decline. Recently, there has been a growing interest in the adoption of multicomponent interventions, usually incorporating nutrition and physical activity strategies targeted to age-related risk conditions like frailty and sarcopenia (1). The protocol published by Low et al. (2) is based on a 4-month program including a combination of group exercise (1 hour, once a week) and the prescription of homebased exercises, together with group-based educational seminars (i.e., six sessions) on nutrition. As outlined in the manuscript, the beneficial effects of nutrition and physical activity as strategies to prevent and manage frailty are well established. However, a challenge in delivering nutritional education programs to older people is represented by the difficulty in motivating persons at acquiring new dietary habits because of multiple reasons (e.g., sociocultural, economic, or clinical issues). The personalization of the interventions may represent an effective strategy to promote these changes (3). assessment able to capture the many aspects of the individual's health (4).

This approach has been carried out in the Sarcopenia and Physical fRailty IN older people: multicomponent Treatment strategies (SPRINT-T) project, including a randomized controlled trial aimed at preventing mobility disability in older people with physical frailty and sarcopenia. The SPRINT-T intervention indeed consisted of a multicomponent interventions designed with a longterm structured physical activity and a personalized nutritional intervention, supported by information and communication technology (5). In SPRINT-T, the nutritional intervention was designed to maximize the benefits of physical activity (Table 1), but took into special account the individual's preferences and resources. A long-term (i.e., 2-3 years) tailored nutrition counseling like that of SPRINT-T can promote a long-lasting behavioral change.

Table 1

Intervention program in the SPRINT-T trial

\begin{tabular}{lll}
\hline Nutritional intervention* & Physical activity program & ICT \\
\hline - 3-day dietary record (to monitor adherence, & $\bullet$ Physical activity classes twice per week including \\
compliance, and personalize recommendations) & $\begin{array}{l}\text { aerobic, strength, flexibility, and balance training } \\
\text { - Energy intake of } 25-30 \mathrm{Kcal} / \mathrm{Kg} \text { of } \mathrm{BW}\end{array}$ \\
$\begin{array}{l}\text { - Protein intake of } 1.0-1.2 \mathrm{~g} / \mathrm{Kg} \text { of BW } \\
\text { - Vitamin D supplementation (if serum levels below } 30\end{array}$ \\
$\mathrm{ng} / \mathrm{mL}$ or $75 \mathrm{nmol} / \mathrm{L})$
\end{tabular}

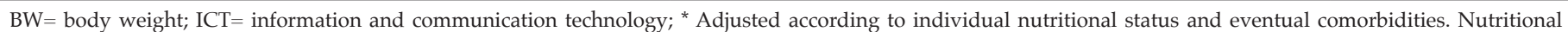
targets could be achieved through supplements if necessary.

Older persons present a marked heterogeneity and high clinical complexity (e.g., presence of comorbidities, mutually interacting syndromes, polypharmacy, differences in physical activity levels). Thus, it seems obvious that nutritional interventions in older people should start from a comprehensive, multidimensional

1. Department of Clinical and Community Sciences, University of Milan, Milan, Italy; 2. Geriatric Unit, IRCCS Istituti Clinici Scientifici Maugeri, Milan, Italy

Corresponding Author: Dr. Domenico Azzolino - Department of Clinical and Community Sciences, University of Milan Via Camaldoli, 64, 20138 Milan (Italy) phone: +390250725218 mail: domenico.azzolino@unimi.it
Unfortunately, in a clinical world driven by the tendency to overdiagnosis and overtreatment, clinicians are still excessively focused on diseases and do not adequately consider the importance of functions. Thus, not surprisingly, the promotion of lifestyle modifications in the prevention/management of frailty and sarcopenia (but likely for any other condition) remains marginal and is too often neglected (6). Instead, especially for chronic conditions and/or in the limited availability of disease-modifying pharmacological treatments, physical exercise and nutrition play a pivotal role and should be considered at the same level of "traditional" medications. 
It is urgent that clinicians should be formally trained at the individual-tailored prescription of physical exercise protocols and dietary recommendations. Only in this way it will be possible to 1) successfully address the older person's priorities, and 2) translate into real life and implement on a large scale the research findings coming from trials testing multicomponent interventions.

Funding: This research received no external funding.

Conflicts of Interest: The authors declare no conflict of interest.

\section{References}

1. Vellas B, Sourdet S. Prevention of Frailty in Aging. J Frailty Aging. 2017;6(4):174-7.

2. Low WL, Sultana R, Huda Mukhlis AB, Ho JCY, Latib A, Tay EL, et al. A non-controlled study of a multi-factorial exercise and nutritional intervention to improve functional performance and prevent frailty progression in community-dwelling pre-frail older adults. J Aging Res Lifestyle. 2021;10:1-7.

3. Ordovas JM, Ferguson LR, Tai ES, Mathers JC. Personalised nutrition and health. BMJ. 2018 Jun 13;361:bmj.k2173.

4. Volkert D, Beck AM, Cederholm T, Cruz-Jentoft A, Goisser S, Hooper L, et al. ESPEN guideline on clinical nutrition and hydration in geriatrics. Clin Nutr Edinb Scotl. 2019 Feb;38(1):10-47.

5. Marzetti E, Calvani R, Landi F, Hoogendijk EO, Fougère B, Vellas B, et al. Innovative Medicines Initiative: The SPRINTT Project. J Frailty Aging. 2015 Dec 1;4(4):207-8.

6. Cesari M. The frailty phenotype and sarcopenia: Similar but not the same. AGING Med. 2019;2(2):97-8. 